\section{La Révolution française}

Cahiers de l'Institut d'histoire de la Révolution française

15 | 2018

Régimes de la propriété, entre l'ancien et le nouveau

\title{
Émigration et gestion des plantations pendant la liberté générale en Guadeloupe (1794-1802)
}

\section{Frédéric Régent}

\section{(2) OpenEdition}

\section{Journals}

Édition électronique

URL : http://journals.openedition.org/lrf/2187

DOI : 10.4000/Irf.2187

ISSN : 2105-2557

\section{Éditeur}

IHMC - Institut d'histoire moderne et contemporaine (UMR 8066)

\section{Référence électronique}

Frédéric Régent, «Émigration et gestion des plantations pendant la liberté générale en Guadeloupe (1794-1802) », La Révolution française [En ligne], 15 | 2018, mis en ligne le 13 décembre 2018, consulté le 21 décembre 2020. URL : http://journals.openedition.org//rf/2187 ; DOI : https://doi.org/10.4000//rf. 2187

Ce document a été généré automatiquement le 21 décembre 2020.

(c) La Révolution française 


\title{
Émigration et gestion des plantations pendant la liberté générale en Guadeloupe (1794-1802)
}

\author{
Frédéric Régent
}

1 Depuis le début de la colonisation en 1635, les colons français ont développé en Guadeloupe, une économie de plantation fondée sur la production de denrées d'exportation. Dans les Antilles françaises, la plantation, appelée "habitation", est la structure économique de base. L'habitation est une exploitation agricole comprenant son fonds de terre, ses bâtiments, ses esclaves et son bétail. La plupart des habitations sont spécialisées dans la production d'une denrée. À la fin du XviII ${ }^{e}$ siècle, les exploitations les plus importantes sont les manufactures à sucre, dont certaines ont une valeur dépassant le million de livres tournois sous l'Ancien Régime. Les années 1789-1792 sont marquées par la prise du pouvoir par les manufacturiers du sucre, qui dominent l'assemblée coloniale aristocratique. De septembre 1792 à décembre 1792, ils placent la Guadeloupe en situation de sécession vis-à-vis de la métropole en ne reconnaissant pas les envoyés de celle-ci, après la chute de la monarchie le 10 août 1792. Ils se heurtent au camp patriote, qui défend les intérêts du commerce avec la métropole et qui recrute ses partisans parmi les marins, les soldats, les marchands et les professions libérales. En janvier 1793, les patriotes prennent leur revanche, avec la proclamation de la République en Guadeloupe. Ce sont désormais les citadins et les petits ou moyens planteurs qui dominent la vie politique. Très peu d'aristocrates émigrent, toutefois beaucoup sont inquiets des nouvelles institutions républicaines. En avril 1794, la Guadeloupe républicaine est attaquée par les Britanniques. Les grands propriétaires accueillent favorablement cette invasion et la plupart des républicains qui participent à la défense de l'île sont faits prisonniers et déportés de l'île. Beaucoup parviennent à rejoindre la France. L'île est placée sous la domination des Britanniques, qui s'appuient sur les grands propriétaires. Le 4 février 1794, la Convention nationale abolit l'esclavage. Un corps expéditionnaire dirigé par le commissaire civil Victor Hugues est chargé d'appliquer la mesure en Guadeloupe, où il débarque le 3 juin. Le corps expéditionnaire parvient à reprendre la colonie, après sa victoire sur des troupes anglo-royalistes. La plupart des grands 
propriétaires prennent la fuite. En 1801-1802, les émigrés sont rappelés. L'objet de cet article est de s'intéresser aux émigrés de la Guadeloupe et à la gestion de leurs biens pendant leur absence et à leur retour.

2 Si les émigrés ou réfugiés de Saint-Domingue ont fait l'objet de nombreuses études ${ }^{1}$, la question de l'émigration depuis la Guadeloupe a été assez peu étudiée ${ }^{2}$. Les situations des deux colonies sont très différentes. Alors que, à Saint-Domingue, les émigrés s'enfuient de manière définitive après la défaite du corps expéditionnaire commandé par Leclerc, puis Rochambeau en 1802-1803, en Guadeloupe, au même moment, l'ordre ancien est rétabli et les émigrés reviennent dans la colonie. La Guadeloupe est le seul exemple colonial français d'un tel retour des émigrés. Si, en métropole, les biens des émigrés sont confisqués, puis vendus sous forme de biens nationaux, aucun bien des planteurs émigrés n'est cédé. Il n'existe pas d'étude empirique sur les émigrés des colonies. En effet, les monographies portent sur les lieux d'arrivée (Cuba, Jamaïque, États-Unis, Louisiane, Martinique, France) des émigrés, et le plus souvent sur une ville (La Havane, La NouvelleOrléans, Saint-Pierre en Martinique, Nantes).

3 Les sources formées par les listes des émigrés, conservées dans le dépôt des papiers publics des colonies, renseignent sur leur identité, mais ne donnent pas d'information sur la région d'émigration. Toutefois, la correspondance administrative des autorités coloniales, les registres notariés conservés aux Archives départementales de la Guadeloupe et l'état-civil éclairent sur les zones de refuge des émigrés. En effet, une personne partie en émigration peut donner naissance à un enfant, se marier ou mourir dans la zone d'accueil. Les actes notariés évoquent parfois l'émigration. Les autorités coloniales indiquent les principales zones de refuge des émigrés. Les listes d'émigrés sont établies par les municipalités par commune en 1796. Les noms des chefs de famille et des membres de leur famille sont mentionnés. Ces listes sont plutôt fiables, car elles peuvent être recoupées avec les deux recensements nominatifs ${ }^{3}$ effectués en Guadeloupe en 1796 et 1797. Il y a très peu de distorsion entre la liste des émigrés et la liste des habitations placées sous séquestre. L'analyse exhaustive des sources (listes d'émigrés, recensements de 1796 et 1797, état-civil, actes notariés) a été faite pour la commune d'Anse-Bertrand. Celle-ci est représentative des espaces produisant à la fois du sucre et une denrée d'exportation secondaire (le coton). Ce croisement des sources permet d'identifier à la fois les émigrés et leurs propriétés. Le récit d'Auguste Lacour peut servir de source ${ }^{4}$. Cet historien du milieu du XIX ${ }^{e}$ siècle, qui s'inscrit dans une historiographie contrerévolutionnaire, a connu des témoins de la période révolutionnaire. Il est intéressant de mettre en perspective son récit des événements avec celui de la correspondance officielle des administrateurs coloniaux ${ }^{5}$.

L'objet de cet article est d'aborder la question de la gestion des biens des émigrés pendant leur absence plutôt que d'étudier leur zone de refuge, question qui nécessite un travail considérable de dépouillement d'archives. L'enjeu est de saisir les raisons de l'absence de mise en vente des biens des émigrés en Guadeloupe et de comprendre ce que deviennent leurs propriétés de 1794 à 1802, pendant la période dite de "liberté générale » qui correspond à leur absence. Après avoir analysé le phénomène de l'émigration en Guadeloupe, il sera procédé à l'étude de la gestion de leurs biens et aux modalités de leur retour. 


\section{Une émigration touchant surtout les grands planteurs}

5 Lors de la reconquête républicaine de la Guadeloupe de juin à décembre 1794, quatre cents à un millier de combattants de l'armée anglo-royaliste sont tués au combat ou exécutés après avoir été faits prisonniers. Il s'agit plutôt de jeunes hommes appartenant à des familles de manufacturiers du sucre et des enfants illégitimes métissés des grands propriétaires. Victor Hugues estime que les libres de couleur composent «la majeure partie des forces anglaises » et, lors de la conquête britannique en avril 1794, ils avaient « abandonné les postes importants dans les moments les plus critiques pour s'incorporer dans l'armée ennemie $"^{6}$. À ces morts à la suite des combats, s'ajoutent vingt-sept guillotinés entre le 12 septembre et le 25 octobre 1794, à Pointe-à-Pitre et au Gosier ${ }^{7}$. Le nombre de morts liés à la collaboration avec l'occupant britannique se situe autour de cinq cents, soit environ $4 \%$ de la population blanche ou réputée blanche de l'époque.

6 La reconquête républicaine provoque la panique des familles de grands planteurs qui avaient pris le parti de l'assemblée coloniale aristocratique entre 1789 et 1792 et avaient déjà été inscrits sur une liste de proscription le 11 mars 1794 par les Républicains. Cette liste concerne quatre cent quarante-huit personnes, pour la plupart des chefs de familles, "prévenues de délits contre-révolutionnaires ${ }^{8}$. Selon Lacour, les proscriptions concernaient «tous ceux qui avaient un nom ou quelque chose». Deux cents quatrevingt-onze des proscrits sont qualifiés de planteurs, dont six seulement de petits planteurs. Les grands planteurs représentent les deux-tiers des proscrits de ce groupe. Sont également présents des cadres de l'administration (directeur du domaine, receveur $\mathrm{du}$ domaine, visiteur du domaine) ou de l'armée (officiers: treize capitaines, dix lieutenants), de la justice (conseiller au Conseil supérieur, juge). Parmi ces hommes, quatre-vingt-six sont qualifiés de "nobles", dont vingt-trois "soit disant nobles ", auxquels s'ajoutent deux comtes, deux barons et un vicomte. Parmi ces proscrits, cinquante-six sont mentionnés comme "valets d'aristocrate", dont les petits planteurs, treize économes, deux notaires. Ceux qui sont qualifiés de valets d'aristocrate sont ceux qui, selon les patriotes, ne devraient pas être parmi les aristocrates au vu de leur rang dans la société. Cette liste de proscription est formée de grands planteurs et de leurs clientèles. Face à cette proscription, la plupart des aristocrates restent chez eux. Quarante-quatre sont emprisonnés ${ }^{9}$. Quatorze sont fugitifs et se réfugient dans une autre commune que la leur. Cent vingt sont déclarés émigrés. Lors de l'occupation britannique d'avril à décembre 1794, ces hommes sont revenus en Guadeloupe et ont activement collaboré avec les occupants en participant à l'administration ou en faisant partie de la force armée. Il est aisé de comprendre, dès lors, la panique que suscite la reconquête républicaine de l'île. Lacour, qui peut s'appuyer sur le témoignage de contemporains ayant vécu les événements, les relate de la manière suivante :

On annonçait que Victor Hugues arrivait à marches forcées avec son armée victorieuse, et qu'il avait fait connaître son intention d'envoyer à la guillotine tous ceux qui ne s'étaient pas montrés franchement républicains. Chacun ne songea qu'à une prompte fuite. Chaque famille se mit à réunir ce qu'elle avait de plus précieux pour courir s'embarquer. [....] on ne voyait dans les rues que des femmes, portant dans leurs bras ceux de leurs enfants qui ne pouvaient les suivre en marchant, et des hommes ayant sur la tête des paquets de ce qu'ils avaient pu rassembler à la hâte : tous fuyaient devant l'armée républicaine, que l'on croyait proche, comme on fuit devant un fléau immédiatement menaçant ${ }^{10}$. 
7 Selon Lacour, mais aussi Thouluyre Mahé, beaucoup de blancs ont émigré à cause de «l'appréhension des scènes d'horreurs que faisait craindre la licence effrénée qui eut lieu parmi les noirs lors de la publication de la liberté générale ». D’après l'auteur, « le tableau effrayant de ce qui s'est passé à Saint-Domingue » a entraîné la fuite de nombreux blancs et réputés blancs de la Guadeloupe ${ }^{11}$. Les listes d'émigrés ne mentionnent pas les dates de ces "départs ", ou du moins de la constatation de leur absence physique. Toutefois, la correspondance de Victor Hugues, le récit de Lacour et quelques allusions chez les notaires indiquent des départs qui se font au moment de la reconquête républicaine de l'île, entre juin et décembre 1794. Un acte notarié d'Anse-Bertrand mentionne que, en « juin 1794, époque de la reprise de la Guadeloupe sur les Anglais » a eu lieu « la sortie de l'île de la grande majorité des colons $»^{12}$.

8 Ces émigrés sont qualifiés de royalistes dans les sources. Beaucoup d'entre eux ont contribué, activement ou passivement, à la Contre-Révolution de la Contre-Révolution (septembre-décembre 1792) et de l'occupation britannique (avril-décembre 1794). Ils ne sont pas forcément tous royalistes, mais sont considérés comme tels par les Républicains radicaux menés par Victor Hugues. Il faut noter que la période qui va de janvier 1793 à avril 1794 a été marquée par des affrontements politiques entre Républicains radicaux (qualifiés de sans-culottes) et modérés (qualifiés de fédérés) ${ }^{13}$. Ces derniers craignent aussi des représailles, étant assimilés aux yeux des premiers comme des « royalistes ».

Des listes comportant des dizaines de noms dans chacun des vingt-six cantons du département révèlent l'ampleur des départs ${ }^{14}$. A la différence de la liste du 7 mars 1794, citée plus haut, tous les membres de la famille sont désormais mentionnés. À Goyave, soixante-trois blancs sont inscrits sur la liste des émigrés ${ }^{15}$ alors que la commune n'en compte que cent trente, d'après le recensement de $1790^{16}$, et n'en a plus que treize en l'an IV $[1794-1795]^{17}$. À Sainte-Anne, on dénombre deux cent vingt personnes émigrées et la population blanche passe de 739 à 530 de 1790 à 1796. En Guadeloupe, la population recensée comme blanche passe ainsi de 13969 en 1790 à 8904 en 1795, que ce soit par mort, déportation, émigration ou absence ${ }^{18}$. Le chiffre total des émigrés se situe à 2925 , selon les listes d'émigrés de la Guadeloupe, dont 127 veuves, 371 femmes mariées, au moins 658 enfants, 85 autres membres de la famille (mère, belle-mère, gendre, bru, pupille, neveu, frère, sœur, belle-sœur), dont ceux qui dans les listes sont inscrits comme accompagnés par leur famille ou leurs enfants, sans précision de nombre (64 cas). À cela s'ajoutent 9 autres personnes (économe ${ }^{19}$...). Il faut ajouter aussi les déportations et les décès des républicains en 1794.

Le nombre de propriétés confisquées est en adéquation avec celui des familles émigrées de maîtres. Les tableaux récapitulatifs effectués par l'administration coloniale renseignent sur la nature des plantations possédées par les émigrés.

Tableau: Habitations séquestrées à la fin de $1794^{20}$ 


\begin{tabular}{|l|l|}
\hline Culture & Habitations séquestrées à la fin de 1794 \\
\hline Sucre & 288 \\
\hline Café & 285 \\
\hline Coton & 70 \\
\hline
\end{tabular}

Tableau : Habitations séquestrées au $1^{\text {er }}$ nivôse an IX [22 décembre 1800]21

\begin{tabular}{|l|l|l|l|}
\hline Culture & Habitations séquestrées & Total des habitations & Proportion \\
\hline Sucre & 299 & 378 & $79 \%$ \\
\hline Café & 273 & 1320 & $21 \%$ \\
\hline Coton & 133 & 826 & $16 \%$ \\
\hline Fourrage & 18 & 39 & $46 \%$ \\
\hline Ensemble & 723 & 2563 & $28 \%$ \\
\hline
\end{tabular}

Tableau : Taille moyenne de la main-d'œuvre selon le type d'habitation (échantillon effectué à partir des recensements de 1796 et 1797 ) 22

\begin{tabular}{|l|l|}
\hline Culture & Nombre moyen d'anciens esclaves attachés aux habitations \\
\hline Sucre & 122,1 \\
\hline Café & 20,9 \\
\hline Coton & 8,5 \\
\hline Vivres & 2,9 \\
\hline
\end{tabular}

11 Plus des trois quarts des propriétaires des sucreries partent en émigration, un cinquième de ceux des caféières et un dixième à un sixième de ceux des cotonneries. En mettant en perspective ces données avec le nombre d'anciens esclaves attachés aux habitations selon leur production, plus les plantations ont d'esclaves avant l'abolition et plus la propension de leurs propriétaires à émigrer est élevée. Ceci corrobore ce qui a été observé plus haut : les petits et moyens planteurs n'ont pas participé à l'assemblée coloniale contrerévolutionnaire et ont rarement occupé des fonctions de commandement dans l'administration pendant l'occupation britannique.

12 Selon le recensement de 1796, 24 des 25 propriétaires de sucreries ont émigré, soit $96 \%$. Le seul manufacturier de sucre qui ne soit pas inscrit sur la liste des émigrés est Pierre Nicolas Desbonne Vannier, qui est parti en France en 1790, avant la Contre-Révolution (septembre-décembre 1792) et l'occupation britannique (avril-décembre 1794), et qui est revenu en $1796^{23}$. La plupart des producteurs de coton ou de vivres sont restés. Seulement 
3 des 46 propriétaires de ce type d'habitation émigrent, soit moins de $9 \%$. Nous avons relevé l'ensemble des émigrés de l'Anse-Bertrand d'après deux listes. Sur la première figurent 135 émigrés $^{24}, 136$ sur la seconde ${ }^{25}$. En 1790, le dénombrement de la population de l'Anse-Bertrand fait état de 345 blancs. En 1795, il n'en reste plus que 228 dans la commune. C'est donc environ un tiers des blancs et réputés blancs qui sont partis. Chaque grand propriétaire part avec sa femme, ses enfants, éventuellement ses petits-enfants. L'analyse des émigrés d'Anse-Bertrand révèle des départs qui se font en famille. Tous les propriétaires de sucreries sont apparentés. À ces derniers, s'ajoutent les propriétaires des principales cotonneries qui leur sont d'ailleurs eux-mêmes apparentés, à la différence des planteurs cotonniers qui restent et qui, eux, appartiennent à un autre réseau familial. À l'Anse-Bertrand, dans les plantations dont les propriétaires émigrent, la moyenne du nombre d'anciens esclaves possédés est, selon le recensement de 1796, de 132, contre 8,5 pour ceux qui restent. Ils sont accompagnés d'esclaves, mais aussi parfois de leur économe. Quelques résidents du bourg ont également émigré : quatre artisans, trois religieux, un négociant et un marin, Il y a parmi eux quelques libres de couleur.

Les émigrés s'enfuient à bord de leurs propres embarcations ou sur des navires britanniques. En outre, dans chaque bourg des quartiers de la Guadeloupe, il y a une ou plusieurs entreprises de pirogue qui servent à transporter les barriques de sucre, les sacs de café ou de coton des plantations aux ports d'exportation que sont Pointe-à-Pitre et Basse-Terre. Ainsi, Étienne Ruillier Duclerc (1763-1820), membre d'une famille de manufacturiers du sucre, est propriétaire en 1804 de deux magasins au bourg de l'AnseBertrand, de deux pirogues et de neuf esclaves marins ${ }^{26}$. Les possibilités de fuite sont donc nombreuses.

Ils se réfugient dans les colonies britanniques voisines: la Dominique, Antigua, Montserrat. Selon un royaliste, Roseau est peuplé d'émigrés de la Guadeloupe ${ }^{27}$. La Martinique occupée par les Britanniques constitue un autre refuge privilégié pour les émigrés. Certains se dirigent vers l'île suédoise de Saint-Barthélemy. L'exemple d'AnseBertrand montre la prépondérance de la Martinique et des îles très proches de la commune que sont Antigua et Montserrat. Les phénomènes migratoires se font donc majoritairement dans un environnement géographique proche. C'est également le cas à Saint-Domingue. Selon une source du Havre mise en évidence par Agnès Renault, la moitié des «blancs » ont péri, un quart est parti en France et un quart s'est dispersé dans le monde ${ }^{28}$. Parmi ceux qui sont allés ailleurs qu'en métropole, un certain nombre s'est installé dans les Antilles britanniques, aux États-Unis ou dans les colonies espagnoles (Louisiane, Cuba, Santo Domingo). Une thèse récente montre que $7 \%$ des Français naturalisés à la Nouvelle-Orléans, de 1817 à 1820, viennent des Antilles, essentiellement de Saint-Domingue ${ }^{29}$.

15 La vie de ces émigrés est peu renseignée par les sources utilisées, il faudrait dépouiller les archives notariées de la Martinique pendant la période révolutionnaire pour en savoir davantage. Un acte notarié concernant Perrine Andreze Douillard la Bertaudière, épouse successive de Guillaume Paviot (1748-1774) puis d'Henry Gaugy, tous deux planteurs à l'Anse-Bertrand, renseigne sur ses moyens de subsistance en Martinique: «Lors de l'émigration, Madame Gaugy a emporté et emmené avec elle des objets de la masse tels que de l'argenterie qu'elle a vendus et des esclaves qu'elle a loués ${ }^{30}$ ». La location des esclaves a constitué un moyen de subsistance pour ces émigrés. En raison du nombre important d'esclaves partis avec leurs maîtres, ces locations ont pu représenter un apport monétaire substantiel. Selon le même acte notarié, les époux Gaugy « songèrent à réparer 
leurs malheurs par l'entreprise d'un commerce qui prospérait jusqu'au décès de Monsieur Gaugy arrivé en 1801 ». La veuve Gaugy fait procéder à l'inventaire en février 1801 par maître Lecamuel. Ce document fait état d'un actif de $108037 £$ en Martinique ${ }^{31}$. Cette reconversion dans le commerce s'avère fructueuse. Certains émigrés se marient à des familles illustres de la Martinique, à l'instar de Pierre Guillaume Ruillier Beauport (1773-1829), qui épouse, au Fort-Saint-Pierre en 1797, Anne Calixte Tascher de la Pagerie, la cousine germaine de Joséphine.

16 Les départs en émigration se font vers des îles sous contrôle britannique, mais aussi aux États-Unis. Les émigrés ont des contacts par des relations commerciales avec la Martinique, les îles britanniques ou les États-Unis, dans le cadre de la contrebande que pratiquent les planteurs avec les capitaines de navires anglais ou américains. À la différence de Saint-Domingue, où les départs de colons se font en fonction des aléas politiques de 1791 à 1804 , ceux de la Guadeloupe se concentrent dans la période de reconquête républicaine de juin à décembre 1794. À Saint-Domingue, les départs débutent dès les premiers affrontements entre factions, les révoltes d'esclaves et de libres de couleur en 1791, et ne deviennent substantiels qu'au moment de l'évacuation britannique (1796-1798) et de la guerre entre Rigault et Toussaint Louverture (1799-1800). De plus, l'orientation politique de ceux qui partent est variable, il peut s'agir au gré des événements de républicains ou de royalistes. En Guadeloupe, ceux qui s'exilent sont royalistes ou réputés tels, en vertu de convictions politiques considérées comme trop peu « républicaines ».

\section{La gestion des propriétés des émigrés pendant la liberté générale}

En vertu du décret sur les biens des émigrés dans les colonies françaises du 25 août 1792, appliquée en Guadeloupe à partir du 9 avril 1793, les biens des émigrés sont séquestrés au profit de la République. Cette loi est la déclinaison coloniale du décret du 27 juillet 1792 qui ordonne la confiscation et la vente au profit de la Nation de tous les biens mobiliers et immobiliers des Émigrés ${ }^{32}$. Avant l'occupation britannique d'avril 1794, il y a cependant peu de confiscations, car peu de départs en émigration. De plus, de janvier 1793 à avril 1794, le gouverneur de la Guadeloupe Victor Collot est un modéré proche des Brissotins, nommé par eux au moment où ils dominent encore la Convention et qui est plutôt conciliant avec les grands planteurs. Toutefois, quelques habitations appartenant à des chefs du parti contre-révolutionnaire sont confisquées. Les sources notariées, lacunaires pour cette période, identifient quatre d'entre elles ${ }^{33}$. Dans le même temps, les habitations appartenant au clergé sont confisquées au profit de la colonie ${ }^{34}$ en conséquence de la nationalisation des biens du clergé. Alors que la loi du 25 août 1792 dispose que « les biens que possèdent, dans les Colonies faisant partie de l'Empire, les Français notoirement émigrés seront saisis \& vendus au profit du Trésor public, le prix en revenant, servir à l'indemnité due à la Nation ${ }^{35} »$, cette première vague de confiscations n'entraîne pourtant aucune vente, les possibilités de vendre à un bon prix n'étant pas réunies. Les acquéreurs potentiels, qui sont les plus riches, sont menacés de proscription ou de mort et peu enclins à investir dans un tel contexte. La guerre qui commence avec la Grande-Bretagne va entraver l'exportation des denrées produites. De plus, la législation interdit la division des manufactures à sucre. Les autorités républicaines, ne souhaitant pas brader à vil prix 
ces exploitations, préfèrent les placer sous séquestre. Il faut ajouter que ces habitations permettent à la colonie de disposer de revenus.

18 Le 13 juillet 1794, alors qu'il vient de faire la reconquête de la moitié de la Guadeloupe, Victor Hugues « requiert la municipalité du Moule de faire séquestrer les biens et traiter en émigrés tous les lâches qui ont fui à Saint-Barthélemy [...], les enjoint en outre de séquestrer les biens de tous ceux qui se sont enfuis; et de les faire arrêter s'ils rentrent le tout sur sa responsabilitée ${ }^{36}$. La décision concerne alors vingt-deux planteurs de la commune. Saint-Barthélemy est alors une colonie de la Suède qui est restée neutre avec la France, si bien que les fugitifs ne peuvent en principe être accusés d'intelligence avec l'ennemi. C'est la raison pour laquelle Victor Hugues vise en particuliers les planteurs partis pour cette île. L'arrêté ne donne pas de précisions sur les modalités de séquestration. Lorsque toute la colonie est reconquise, un arrêté du 22 novembre 1794 annonce la séquestration des biens de l'ensemble des émigrés ${ }^{37}$.

À la fin de 1794, 288 habitations sucrières (79\% de ces habitations), 285 caféières (21 \%), 70 cotonnières et vivrières ( $16 \%$ ) et 200 maisons dans les 28 communes de la colonie sont séquestrées suite à l'émigration de leurs propriétaires ${ }^{38}$. Le mouvement de confiscation des biens semble se poursuivre car 704 habitations le sont en l'an V [1796-1797] (300 sucreries, 6 sucreries et caféières, 2 sucreries et cotonneries, 2 sucreries, caféières et cotonneries, 125 caféières, 164 caféières et cotonneries, 13 cotonneries de peu de valeur, 14 vivrières et 77 abandonnées) $)^{39}$, et 723 habitations au 22 décembre 1800 (299 sucreries, 273 caféières, 133 cotonneries, 18 en fourrage) ${ }^{40}$. Une récapitulation du 18 octobre 1800 fait état de 983 habitations séquestrées et mises en valeur par 39252 nouveaux citoyens ${ }^{41}$. L'augmentation du nombre d'habitations séquestrées s'explique par la politique de Victor Hugues, jugée abusive en matière d'inscription sur les listes d'émigrés par l'historien Lacour, qui a bien connu des témoins de l'époque ${ }^{42}$. Le 22 février 1795, un arrêté ordonne «à toutes personnes absentes de la colonie par suite de déportation des gouvernements antérieurs, ou pour toute autre cause, de rentrer dans le délai de deux mois, sous peine d'être déclarées émigrées et d'avoir leurs biens confisqués ${ }^{43}$ ». L'administration doit veiller à distinguer les émigrés des absents. En effet, un certain nombre de colons sont en dehors de la colonie pour des raisons familiales ou économiques et ne peuvent y revenir en raison de la guerre. Victor Hugues les considère ainsi comme des émigrés, ce qui permet de doubler le nombre de propriétés confisquées.

Lorsque nous disons 638 habitations, nous n'entendons parler que de celles sur lesquelles il y eut main mise aussitôt l'arrivée de Victor Hugues, car ce nombre ne tarda pas à être doublé. En effet, à la Guadeloupe, presque toutes les confiscations ont eu lieu de juin à décembre 1794. Or, en l'année 1801, malgré un certain nombre de restitutions faites tant par Desfourneaux ${ }^{44}$ que par les agents Jeannet, Laveaux et $\mathrm{Baco}^{45}$, on comptait encore 1005 habitations confisquées. C'étaient les plus considérables du pays car, ici comme en France, c'est parmi les grands propriétaires que l'on compta le plus grand nombre d'émigrés ${ }^{46}$.

Les propriétés des personnes réputées émigrées sont signalées par un écriteau " propriété nationale». Le panneau "propriété séquestrée» concerne celles pour lesquelles les propriétaires absents n'ont pas pu fournir de certificat de civisme ${ }^{47}$. Les confiscations, qui concernaient d'abord ceux qui étaient convaincus d'intelligence avec l'ennemi et d'opinions royalistes, s'étendent à des « suspects » supposés royalistes, faute de garanties tangibles de leur républicanisme. Victor Hugues applique ainsi le décret du 8 ventôse an 2 [26 février 1794], qui place sous séquestre les biens des suspects au profit de la République ${ }^{48}$. 
21 Pour assurer la gestion de chaque propriété nationale ou séquestrée, l'administration municipale désigne un homme appelé séquestre. Selon Lacour, le choix de ces séquestres est «déterminé plus par le sans-culottisme que par les autres garanties ${ }^{49}$ ». Sur un échantillon de 171 séquestres, 138 sont blancs ou réputés blancs, les autres sont de couleur. La plupart des séquestres sont d'anciens libres blancs ou de couleur, il y a cependant quelques anciens esclaves désignés séquestres par les administrations municipales. Sur les habitations concernées à l'Anse-Bertrand, certains séquestres appartiennent au milieu des petits planteurs de vivres, à l'instar de Pierre Dupré Rivière pour la sucrerie Labazordière ou François Daube pour la cotonnerie de la famille Hyance. Denis Drouault, séquestre de l'habitation nationale Labourgerie, appartient à une famille de manufacturiers du sucre. Il vit en ménage avec une femme noire, Scholastique, avec laquelle il a eu Euphrosine, une enfant métissée. Jean-Baptiste Le Curieux Clerville, issu d'une famille d'habitants de la Martinique, est un réfugié républicain de cette île ${ }^{50}$. Son engagement politique lui permet d'accéder à la charge de séquestre. Les séquestres sont choisis parmi ceux qui sont proches des administrations municipales en place, ellesmêmes désignées par Victor Hugues. Ce dernier, nommé commissaire civil à la Guadeloupe par la Convention montagnarde, s'appuie sur ceux qui étaient qualifiés de sans-culottes, pendant le gouvernement de Collot (janvier 1793-avril 1794).

Les séquestres n'ont pas le droit de punir les citoyens attachés aux habitations nationales. Ils doivent dénoncer leur manque de travail, leurs vols et c'est ensuite aux municipalités de prononcer des peines. Un arrêté du 8 janvier 1798 rappelle ces dispositions. Victor Hugues se plaint des séquestres qui infligent des peines correctionnelles aux anciens esclaves $^{51}$. Les pratiques violentes de l'esclavagisme se perpétuent donc malgré l'abolition de l'esclavage.

23 Les revenus des habitations confisquées reviennent au budget de la colonie. Les séquestres sont placés sous le contrôle des administrations municipales. Chaque décade, le séquestre doit remettre un rapport sur ce qui s'est passé sur l'habitation nationale. Ce rapport est formé des éléments suivants :

- Quantité de citoyens en général ;

- Travail qu'il a fait ;

- Vivres qu'il a distribués ;

- Naissances de citoyens ;

- Morts de citoyens ;

- Absents qu'il y a \& pourquoi ;

- Naissances de bestiaux;

- Mortalité de bestiaux ;

- Chevaux du dépôt ${ }^{52}$

Ce compte ainsi rendu, le séquestre reçoit sa ration de la municipalité. Le primidi de chaque décade, le maire ou deux officiers municipaux inspectent les sucreries et caféières de la République pour vérifier le compte de chaque séquestre. Selon Lacour, les séquestres, estimant ne pas être assez bien rémunérés pour leur travail, grappillent sur les rations des cultivateurs ou se livrent à des petits larcins ${ }^{53}$. Les cultivateurs font souvent de fausses dénonciations contre les séquestres. Ils les accusent de prendre des vivres de l'habitation pour leur subsistance ${ }^{54}$. Le contrôle de l'administration sur ces plantations séquestrées est d'autant plus tatillon qu'elles constituent pour elle sa seule source de revenus avec les prises de navires par les corsaires, armés sur le budget de la colonie. La quasi-disparition des exportations des denrées coloniales et des importations 
du fait de la guerre a entraîné celle des droits sur l'entrée et la sortie des marchandises qui alimentaient majoritairement le budget de la colonie. Les habitations nationales permettent de payer les membres des administrations municipales. Ainsi, les officiers municipaux et le délégué étant privés de rations, deux habitations nationales sont choisies pour leur fournir farine et laitage ${ }^{55}$.

Si les plantations des émigrés ou des «suspects " sont placées sous le contrôle d'un séquestre, les maisons urbaines appartenant aux émigrés sont quant à elles simplement louées. Les locataires ont même la possibilité de sous-louer ${ }^{56}$. Lacour signale que, au début de l'administration de Victor Hugues, toutes les petites propriétés qui ne font pas assez de revenus pour payer un séquestre sont louées au profit des caisses de la République. C'est le cas, le 11 octobre 1795, des habitations de taille plus importante dans la région de Basse-Terre (Versailles, Thillac, Lemerle, veuve Hugenet, Dampierre, Servule, Boudet, Duvivier, Audinet, Rougemont). Selon Lacour, « Victor Hugues méditait d'étendre à toutes les propriétés séquestrées le système des baux à ferme $\mathrm{e}^{57}$ ».

La non mise en vente des biens placés sous séquestre est confirmée par la loi du 30 septembre 1797, qui dispose que, « aussitôt après la paix générale, le gouvernement fera procéder à l'état des biens nationaux, terrains vagues et indéfrichés qui peuvent exister dans l'île de Saint-Domingue et autres colonies françaises ; il sera procédé successivement à leur vente ${ }^{58}$ ». En creux, la loi interdit la vente des biens des émigrés dans les colonies tant que se poursuit la guerre. La loi concernant l'organisation constitutionnelle des colonies du 12 nivôse an VI [ $1^{\mathrm{er}}$ janvier 1798] rappelle cette disposition. Cette loi met en place une régie de tous les biens nationaux dans les colonies, qui est confiée à trois administrateurs, nommés et surveillés par les agents du Directoire. La régie est chargée de veiller à la conservation de toutes les propriétés nationales et d'empêcher surtout que, sans un bail fait à la suite d'une enchère publique, quiconque puisse s'établir sur les propriétés et même sur des terres incultes et non concédées. Les biens nationaux dans les colonies sont donnés à ferme pour un temps qui ne peut excéder cinq années et adjugés publiquement au plus offrant et dernier enchérisseur. Les agents du Directoire, les ordonnateurs, chefs et préposés d'administration, les commandants en chef et officiers généraux dans l'étendue de leur commandement ne peuvent être adjudicataires ni fermiers, par eux ni par l'entremise d'un tiers. Les agents du Directoire sont autorisés à faire réviser les baux à ferme qui auraient pu être passés avant la loi, et à les faire mettre de nouveau à l'enchère, si la République avait été lésée d'un tiers dans le prix des adjudications. Les baux affermés à l'amiable et sans enchères sont déclarés nuls. Les deux tiers du produit net des revenus des habitations séquestrées sur les émigrés sont appliqués aux dépenses publiques. Le dernier tiers est destiné au paiement des créanciers et autres personnes ayant des droits à exercer sur ces biens ${ }^{59}$. Les loyers étant payés en denrées (sucre, coton ou café), les habitations ne produisant aucun des produits précédents sont louées en numéraire. Le bail est payé par tiers tous les quatre mois, le premier paiement s'effectuant le premier mois après l'adjudication ${ }^{60}$.

Le 17 juillet 1799, le successeur de Victor Hugues, l'agent du Directoire Desfourneaux, décide d'affermer les habitations nationales de la Guadeloupe et ainsi d'appliquer la loi du $1^{\mathrm{er}}$ janvier 1798. Celle-ci, en affirmant que les biens des émigrés des colonies ne pourront être vendus qu'à la paix, entérine la pratique qui avait consisté à ne pas les proposer à l'achat. L'utilisation des séquestres avait été une mesure transitoire, en attendant une éventuelle mise aux enchères. La guerre qui dure force le législateur à mettre en place le système de location des biens des émigrés, en attendant de procéder à leur mise en vente, 
dans l'éventualité de la fin de la guerre. L'avantage de la location est l'obtention de revenus fixes.

Un exemple de bail à ferme d'une habitation nationale permet de mieux comprendre le fonctionnement concret de ce nouveau système. Il concerne la caféière Bruno Mercier aux Trois-Rivières. Vingt-deux cultivateurs, dont neuf enfants, y sont attachés. Le terme « attaché » qualifie le lien de dépendance qui existe entre l'ancien esclave et son ancien maître ou son ancienne habitation. Le bail est fixé après enchères à 4800 livres de café par $a^{61}$. Les sources fournissent peu d'informations sur ces locataires d'habitations nationales. Parmi ces derniers, on trouve des négociants, comme Robin ${ }^{62}$, François Robert et Guillaume Costet, qui prennent le 23 juillet 1799 en adjudication le bail à ferme pour cinq ans de l'habitation Fond Cacao à Capesterre, établie en sucrerie pour 74000 milliers de sucre par an. Ils décident de former une société pour l'exploiter ${ }^{63}$. Pierre Nicolas Desbonne Vannier, seul manufacturier du sucre demeurant à l'Anse-Bertrand, est locataire de l'habitation nationale René Ruillier ${ }^{64}$. Parmi les autres locataires relevés, il y a un lieutenant de la compagnie des sapeurs, Jean Joseph Mamon, qui prend un "géreur » pour administrer l'habitation nationale à sa place ${ }^{65}$, et un habitant des Trois-Rivières, Jean Joseph Rommieu, qui se rend adjudicataire d'une plantation de café à Bouillante. Il se plaint d'ailleurs des conditions de l'adjudication. Il constate que les bâtiments sont en plus mauvais état que ne le présentait la description donnée lors de la mise aux enchères et que le nombre de cultivateurs est inférieur à celui qui y était indiqué ${ }^{66}$. Négociants, officiers, habitants républicains, mais aussi européens nouvellement arrivés sont donc les principaux bénéficiaires de la location des biens des émigrés. Or, ces fermiers de la République adoptent les mêmes comportements que les anciens planteurs à l'égard des cultivateurs et notamment le recours à la contrainte ${ }^{67}$.

Le général Desfourneaux se félicite du succès de son système et affirme qu'il a " porté au plus haut prix la location des biens nationaux. [...] Les agents qui ont remplacé le général Desfourneaux paraissent contredire ce résultat. [...] Ces agents disent de plus qu'ils reçoivent journellement un grand nombre de demandes en diminution du loyer des habitations nationales affermées par l'agent Desfourneaux ${ }^{68} \%$. Ils obtiennent des successeurs de ce dernier, le 17 février 1800, la résiliation des baux dont ils trouvent les montants trop élevés et leur remise aux enchères. Les locataires ont dix jours pour demander la résiliation. Ils ne peuvent le faire qu'après avoir payé leur bail. Vingt jours après, il est procédé à une réadjudication des habitations nationales dont les baux ont été résiliés. Une estimation des biens est faite avant la réadjudication ${ }^{69}$.

Comme les acquéreurs de biens nationaux en métropole ${ }^{70}$, l'affermage des habitations nationales guadeloupéennes profite surtout aux élites urbaines (bourgeoisie marchande, armateurs de corsaire, maitres artisans, professions libérales) et aux plus gros propriétaires fonciers. Ainsi, parmi les adjudicataires, se trouvent les quelques manufacturiers du sucre restés dans la colonie et les propriétaires des plus grandes habitations de café ou de coton. Si les séquestres étaient principalement des sansculottes, les locataires sont principalement des républicains modérés, à l'instar du général Desfourneaux, l'agent du Directoire alors chargé de diriger la Guadeloupe.

Du fait de la guerre, qui entrave la commercialisation des denrées d'exportation, plus que du mode de gestion des biens des émigrés et des "suspects ", les superficies cultivées en cannes à sucre, café et coton s'effondrent entre le début de la Révolution et 1799. Tableau : Surfaces cultivées en 1790 et au $7^{\text {er }}$ vendémiaire an VIII [23 septembre 1799] ${ }^{71}$ 


\begin{tabular}{|l|l|l|l|}
\hline Culture & $\mathbf{1 7 9 0}$ & An VIII & Variation entre 1790 et l'an VIII \\
\hline Surface en cannes & 22686 & 7283 & $-68 \%$ \\
\hline Surface en café & 8607 & 5281 & $-39 \%$ \\
\hline Surface en coton & 8766 & 2216 & $-74 \%$ \\
\hline Surfaces en vivres & 11220 & 4330 & $-61 \%$ \\
\hline Ensemble & 51279 & 19648 & $-62 \%$ \\
\hline
\end{tabular}

La très forte diminution des superficies plantées en vivres ne concerne que les parcelles exploitées en commun par les cultivateurs et non leurs jardins particuliers, qui ont pu connaître un certain développement. Le phénomène d'abandon des terres pendant la liberté générale est très important et l'administration coloniale ne le cache pas. L'abandon des terres est la conséquence de différents facteurs : émigration, vagabondage, exode rural et engagement des cultivateurs dans l'armée, mais il est également dû à une baisse de leur productivité et, éventuellement, à une réorientation de leur travail en direction de leurs jardins. La maitrise de l'océan Atlantique par les Britanniques rend très difficile l'exportation des denrées coloniales. Les propriétaires des plantations, séquestres ou locataires, sont peu enclins à produire des denrées devenues difficilement commercialisables. Le recul des surfaces cultivées en denrées coloniales entraîne le recul de la production.

Tableau : Denrées coloniales produites sur les habitations nationales ${ }^{72}$

\begin{tabular}{|l|l|l|l|l|}
\hline Denrées fabriquées & An II & An III & An IV & An V \\
\hline Sucre terré (barrique) & 4299 & 5645 & 5378 & 4468 \\
\hline Sucre terré (tonneau) & - & 3 & 3 & 36 \\
\hline Sucre terré (quart) & - & 4 & 1097 & - \\
\hline Sucre brut (barrique) & 1652 & 3439 & 1877 & 1756 \\
\hline Sucre brut (tonneau) & - & 42 & 18 & 19 \\
\hline Sucre brut (quart) & - & 111 & 64 & - \\
\hline Sirop (galion) & 49810 & 505765 & 562164 & 333642 \\
\hline Rhum (galion) & 8978 & 81876 & 120837 & 89575 \\
\hline Café bonifié (baril) & 2864 & 11323 & 9813 & 10924 \\
\hline Café triage (baril) & 490 & 699 & 77 & 57 \\
\hline Coton épluché (en £) & 65729 & 39920 & 28196 & 19341 \\
\hline
\end{tabular}




\begin{tabular}{|l|l|l|l|l|}
\hline Coton sur pieds (en $\mathbf{~}$ ) & 173252 & 202690 & 115435 & 122352 \\
\hline Cacao (baril) & - & 63 & 37 & 103 \\
\hline
\end{tabular}
après un arrêt presque complet de la production en 1794, imputable aux combats se déroulant sur le sol guadeloupéen, la production se redresse dans les années qui suivent. Il faut noter la très forte hausse de la production de rhum et de sirop, denrées qui intéressent les Américains. Ces derniers produisent notamment des eaux-de-vie à partir des sirops. Le développement de l'activité corsaire sous Victor Hugues peut expliquer une augmentation de la consommation d'alcool. Le rhum est plus facilement commercialisable dans le reste de la Caraïbe, y compris dans le cadre de la contrebande avec les Antilles britanniques. l'abolition de l'esclavage et l'interruption de la traite négrière contribuent aussi à cette baisse. Le départ ou l'absence des propriétaires est, par contre, un facteur plus négligeable dans la baisse de la production. Ainsi, les superficies plantées en coton diminuent dans la même proportion que celles cultivées en cannes à sucre, alors que la plupart des planteurs de coton sont restés, à l'inverse des manufacturiers du sucre.

Les agents du Directoire Baco, Jeannet et Laveaux critiquent le système de location, car la République, qui disposait directement du produit des habitations nationales, dépend désormais de fermiers qui ne la payent pas ou fraudent ${ }^{74}$. Ainsi, certaines habitations nationales ont été louées sans véritable bail régulier comme récompense ${ }^{75}$. Certains fermiers qui payent en sucre ont mis du tuf, du fumier et des corps étrangers dans les barriques $^{76}$. À plusieurs reprises, les administrateurs coloniaux se plaignent de la mauvaise rentrée des fermages des habitations ${ }^{77}$. Il est difficile de déterminer, tant les témoignages sont contradictoires, si le système des locations est plus avantageux financièrement pour les finances de la colonie que celui des séquestres.

Du fait des circonstances de la guerre, mais aussi de l'émigration de la majorité de ceux qui auraient les moyens de les acheter à un prix élevé, la vente des biens des émigrés des colonies, bien que prévue par la loi du 25 août 1792, a été suspendue localement. Ceci a été ensuite entériné par les lois du 30 septembre 1797 et du $1^{\text {er }}$ janvier 1798. Sur le plan local et national, les autorités se sont donc adaptées à la situation. Les avantages de la pratique de la mise sous séquestre entre 1794-1798 sont la nomination directe et la possibilité de les révoquer à tout moment par l'administration de la colonie. Elle assure également que le produit net des habitations revienne à la colonie. L'inconvénient de ce système est l'absence d'investissement. Un séquestre n'a aucun intérêt à investir dans une plantation dont il peut être révoqué à tout moment. De plus, beaucoup de séquestres, $\mathrm{du}$ fait de leurs origines sociales modestes, n'ont pas les moyens financiers pour le faire. La mise en location entre 1798-1802 a exactement les avantages et qualités inverses du système des séquestres.

\section{Le retour des maîtres émigrés}

37 Victor Hugues a mené une politique de mise sous séquestre des biens des planteurs émigrés et absents particulièrement zélée. Lacour estime que le commissaire civil de la 
Convention devenu agent du Directoire, après son mariage avec une femme issue de la plantocratie, le 15 mars 1796, a assoupli sa politique en matière de confiscation $^{78}$. Ses successeurs, les agents Desfourneaux (1798-1799), Jeannet, Laveaux et Baco (1799-1801) ont opéré des restitutions ${ }^{79}$.

Certains propriétaires absents souhaitent en effet rentrer sur leurs habitations. Dès que l'opportunité se présente, ils le font. Nicolas Desbonne Vanier profite du fait d'être parti pour un séjour en France avant la Contre-révolution et l'occupation britannique pour revenir dès 1796 . La loi du $1^{\mathrm{er}}$ janvier 1798 dispose que le séquestre apposé sur les biens d'une personne absente, non portée sur la liste des émigrés des colonies, est levé. Cela permet aux absents d'entamer une procédure de radiation des listes des émigrés. Ainsi, Jacques Pierre Ruillier (1763-1836) revient d'émigration à une date inconnue, mais avant le 29 septembre 1800, date à laquelle il déclare, à l'Anse-Bertrand, la naissance de son fils Charles, surnommé « Monretour »,

Le coup d'état de Bonaparte suscite ainsi des espérances chez les émigrés. Duny, un métropolitain, ancien régisseur à Saint-Domingue qui séjourne en Guadeloupe au début de 1801, évoque ses espoirs : «Les propriétaires, les agriculteurs, les négociants ont été placés entre le poignard et l'échafaud; ils ont été dépouillés, proscrits, assassinés, quelques-uns ont échappé à leurs bourreaux et n'ont cessé de tourner leurs regards vers leur patrie. Ils ont vécu dans les îles voisines où ils ont pu aborder, dans les privations de toute espèce ». Il prend la défense de ces émigrés qui ont fui la mort. Il espère que le régime consulaire installé par le 18 brumaire rétablira l'ordre en Guadeloupe ${ }^{80}$.

Lacrosse prend ses fonctions de capitaine général de la Guadeloupe en mai 1801. Le 3 juin, il annonce que plus personne n'est considéré comme émigré. On lui fait remarquer que les émigrés ont lutté pour l'Angleterre et que, les baux à ferme de leurs biens séquestrés étant pour cinq ans, des problèmes pourraient se poser. Sur cette remarque, Lacrosse suspend sa proclamation ${ }^{81}$. Il donne l'ordre de ne plus rechercher les émigrés qui se présenteraient dans la colonie ${ }^{82}$. Cette politique s'inscrit dans celle du Premier Consul, qui commence par faire cesser toute poursuite à l'égard des émigrés ${ }^{83}$. L'arrêté consulaire du 20 octobre 1800 élimine des personnes des listes d'émigrations, notamment les conjoints et enfants mineurs d'émigrés ${ }^{84}$.

41 Le 28 juin 1801, Lacrosse rappelle en Guadeloupe « les habitants, trop longtemps absents de cette colonie, que la presque certitude d'être victimes des événements révolutionnaires avaient éloignés ». Cette décision provoque un fort mécontentement. Dans la nuit du 28 au 29 juin, toutes les affiches de cette proclamation sont arrachées et lacérées ${ }^{85}$. Sur la foi de la proclamation du 26 juin, beaucoup d'émigrés rentrent cependant en Guadeloupe. « Mais ils n'eurent pas plus tôt vu la situation de la Guadeloupe que plusieurs se hâtèrent de s'expatrier de nouveau, effrayés des malheurs qu'ils croyaient devoir fondre sur la colonie ", affirme Lacour. En effet la politique vexatoire menée par Lacrosse contre les républicains avancés et l'armée de couleur provoque des tensions qui suscitent de fortes inquiétudes. Ainsi, Budan, habitant-propriétaire à l'AnseBertrand, qui avait émigré, écrit ceci :

Le citoyen Lacrosse n'a pas plus de politique que d'humanité. Où sont ses forces pour se permettre toutes les violences qu'il exerce ? Les noirs et les hommes de couleur non propriétaires composent les sept huitièmes de l'armée, et il leur déclare la guerre sans motif ! N'est-il pas à craindre qu'ils ne finissent par se révolter, et qu'ils ne répètent à la Guadeloupe toutes les scènes de dévastation et de carnage dont Saint-Domingue a été si longtemps le théâtre? Je ne vois point de 
sûreté à rester dans un pays gouverné par un tel homme, qui ne trouve pas de milieu entre ses anciens excès et les excès de sa prétendue conversion ${ }^{86}$.

La politique vexatoire de Lacrosse a conduit les officiers de couleur, soutenus par les républicains avancés et les bénéficiaires de la liberté générale, notamment les fermiers des habitations des émigrés et des absents, à le destituer et à le renvoyer de la Guadeloupe en octobre 1801. Un conseil de gouvernement provisoire de la Guadeloupe s'est mis en place. Il défend les intérêts des locataires des habitations confisquées. En mai 1802, le corps expéditionnaire de Richepance détruit la rébellion menée par les officiers de couleur Delgrès et Ignace. Dans le prolongement d'une répression sanguinaire, il rétablit la pratique de l'esclavage en Guadeloupe. Les émigrés de retour profitent du sénatus-consulte du 6 floréal an X [26 avril 1802], qui accorde amnistie pour fait d'émigration à tout individu qui en est prévenu. Pour la métropole, seuls mille émigrés sont maintenus sur les listes. Le retour de ces émigrés s'accompagne de la prestation du serment de fidélité à la Constitution et de n'entretenir, ni directement ni indirectement, aucune liaison ni correspondance avec les ennemis de l'État. Pendant dix années, les émigrés amnistiés sont placés sous la surveillance spéciale du gouvernement, à dater du jour de la radiation, élimination ou délivrance du certificat d'amnistie ${ }^{87}$. Un arrêté de Richepance du 10 thermidor an X [29 juillet 1802] supprime en conséquence toutes les listes d'émigrés. Il remet à ces anciens proscrits toutes les propriétés qui étaient restées libres entre les mains de l'administration, et leur fait abandon du revenu de celles qui étaient affermées. La mesure de Richepance dispense toutefois les émigrés de la Guadeloupe de toute forme de surveillance. Les grands planteurs sont en effet considérés davantage comme des victimes que comme coupables d'émigration. Cette dernière est donc purement et simplement effacée. Seuls ceux qui ont émigré depuis la métropole sont considérés comme tels. En effet, certains propriétaires résidaient en métropole et sont partis en exil depuis celle-ci. Ils sont considérés comme plus coupables que ceux qui sont partis directement de Guadeloupe. Le 3 septembre 1802, un arrêté consulaire ordonne même à tous les colons qui s'étaient absentés pendant la Révolution d'aller reprendre possession de leurs biens dans les plus brefs délais. Il ordonne de résilier tous les baux des habitations, maisons ou magasins des absents ou émigrés de la Guadeloupe. L'arrêté, pour prononcer cette résiliation, se fonde sur le fait que « ces baux avaient été généralement consentis à vil prix ${ }^{88} »$. Un arrêté des consuls du 28 brumaire an XI [19 novembre 1802] confirme cette décision ${ }^{89}$.

43 Alors que la législation métropolitaine ne fait qu'autoriser le retour des émigrés sur le sol français, en Guadeloupe, le retour des émigrés est encouragé par les décisions locales, entérinées nationalement. Les fermiers des habitations nationales sont considérés comme des partisans actifs ou passifs de la rébellion contre Lacrosse. Le rappel des émigrés et des absents permet aux représentants du pouvoir consulaire d'avoir des soutiens, tout en sanctionnant ces fermiers qui ont vu avec satisfaction le renvoi de Lacrosse qui favorisait le retour des émigrés dès 1801 . Richepance et Lacrosse cherchent à renforcer leur pouvoir en s'appuyant sur les émigrés contre les fermiers.

Une autre mesure est prise en faveur des émigrés. Tous les intérêts des capitaux empruntés sont gelés pour la période allant du 4 juin 1794 (Grande-Terre) et du 8 octobre 1794 (Basse-Terre) au 23 septembre 1802. L'arrêté est approuvé par une décision du Conseil d'État du 3 septembre 1803. Boyer-Peyreleau salue la mesure, mais évoque les effets pervers qu'elle suscite : 
Le tempérament adopté était sage et juste envers ceux qui avaient éprouvé de grands malheurs. Il empêchait le bouleversement des propriétés, sans effrayer le crédit, donnait aux planteurs, dispersés pendant l'orage, la faculté de reprendre leurs travaux sur de nouveaux frais, et de trouver, dans leurs prochains revenus, les moyens de se liquider. Mais combien d'entre eux devaient en abuser pour frustrer l'espoir de leurs créanciers, et tromper le vœu du gouvernement ${ }^{90}$ ! colonie, le préfet colonial de la Guadeloupe Lescallier fait paraître un règlement contenant les formalités à suivre pour déterminer les droits des propriétaires et des fermiers. Le propriétaire, dont les droits sont reconnus, reçoit un ordre qui lui restitue sa pleine et entière propriété en lieu et place des fermiers. Muni de cet ordre, il le porte au commissaire du quartier, lequel, après huit jours au plus tard, doit se rendre sur la propriété, assisté de deux planteurs nommés, l'un par le fermier et l'autre, par le propriétaire. Ces planteurs experts, en comparant l'état actuel de l'habitation avec son état lors du bail, fixent l'indemnité à payer soit au propriétaire, soit au fermier. L'indemnité est calculée en fonction de l'augmentation ou de la diminution de la valeur de l'habitation entre le début du bail et la restitution. En effet, il faut noter le mauvais état des habitations récupérées. Les superficies plantées en cannes à sucre, faute de débouchés à l'exportation pendant la liberté générale, ont été fortement diminuées, et les bâtiments peu entretenus. Toutefois, ne doivent pas être mis à la charge des fermiers les déficits survenus par force majeure et par l'effet de la rébellion de mai $1802^{91}$. Sur les 153 habitations que compte Basse-Terre, théâtre principal des combats, 25 ont été complètement incendiées, 72 pillées ou dévastées, une a sauté par l'effet de la poudre ${ }^{92}$.

Ces mesures favorables encouragent le retour des émigrés ${ }^{93}$. Nous estimons que la quasitotalité de ceux qui ont survécu à l'exil reviennent, comme le montre l'exemple de l'AnseBertrand. Tous les habitants de cette commune et leurs familles rentrent ainsi sur leurs terres. L'analyse des actes notariés et des registres d'état civil le démontre. Un acte datant des 13 et 14 mars 1806 précise le déroulement de la procédure de restitution :

Que les planteurs de la Guadeloupe furent rappelés et rentrèrent en 1801 et 1802 mais que leurs propriétés ne leur furent pas rendues par le gouvernement qu'il leur fut seulement permis de traiter avec ceux qui tenaient à bail leurs habitations que le six fructidor an X [30 août 1802] ceux des associés rentrés dans l'île traitèrent avec le locataire de l'habitation et en furent mis en possession. De cette époque, il s'écoula encore quelques tems jusqu'à la levée du séquestre et la pleine restitution de l'habitation, qu'il a fallu depuis réunir titres et papiers dispersés, tâcher de suppléer à ceux égarés ou perdus, lever des expéditions de divers actes et parcourir divers dépôts et archives à cette fin, réunir même une partie des esclaves dispersés durant une longue anarchie ${ }^{94}$.

Ce cas particulier est emblématique de la lenteur avec laquelle s'opèrent les restitutions. Les maîtres de retour sont confrontés à plusieurs problèmes. Ils doivent traiter avec les fermiers qui ont obtenu, par adjudication, le bail de leur habitation pour cinq ans. Mais ces arrangements sont manifestement bien difficiles si l'on en croit le mémoire du général Ménard. Ce dernier estime que, parmi les fermiers, il existe des hommes qui ont pour objectif «l'assassinat des habitants, leur expulsion de la colonie, la rentrée des propriétés sous le système des séquestres ; et les disciples des Barsse et la Girardière, plus heureux le 28 germinal [18 avril 1803] que le 14 vendémiaire [ 6 octobre 1802], ne s'en tiendront pas à ce faible avantage ${ }^{95}$ ». Ménard, qui a remplacé Richepance, à sa mort,

La Révolution française, 15 | 2018 
comme général en chef de l'armée, évoque ici son renvoi de la Guadeloupe par Lacrosse, le 18 avril 1803. Le 6 octobre 1802 fait référence à la révolte de Sainte-Anne déclenchée par trois chefs blancs : Barsse, Millet de la Girardière et Jean Barbet. Pendant la liberté générale, Barsse était commissaire du gouvernement et fermier de l'habitation Gassien, appartenant aux héritiers de Vipart ${ }^{96}$; Millet de la Girardière était un ancien officier français, colon de la Martinique, qui venait d'en être chassé pour ses idées politiques certainement républicaines ; Jean Barbet, natif d'Antenac, en Gascogne est laboureur. Les mulâtres Yves (sans nom de famille), Louis Bureau, Jean Gautier, René Gayan et Louison Bourk, ainsi que les nègres Hippolyte, Édouard et Jean (domestique de Barsse) faisaient partie de la conspiration. Ils parcourent vingt habitations, recrutant des insurgés dans chacune : leur nombre monte à quatre-vingt, dont vingt à cheval. Vingt-trois blancs sont ainsi tués par les insurgés. Il n'est pas précisé s'ils sont des émigrés de retour ${ }^{97}$. La révolte est matée par la garde nationale. Selon Ménard, il y a une forte opposition entre fermiers et émigrés de retour. À ce propos, il déclare : «j'ai considéré la Grande-Terre, comme le champ de bataille des locataires et des propriétaires ${ }^{98}$ ». Le clivage entre fermiers et propriétaires de retour est à la fois politique et social. Les locataires perdent la gestion des manufactures à sucre et retournent à leur position sociale souvent plus modeste (planteurs de café ou de coton, notables des bourgs et des villes). La révolte de SainteAnne a été une tentative avortée de collusion - contre le nouvel ordre des choses, dominé par le pouvoir consulaire et les émigrés - de tous les mécontents : fermiers perdant la jouissance des biens des émigrés, cultivateurs remis en esclavage et débris des rebelles de l'armée de couleur. Ménard estime que la colonie est toujours menacée par au moins trois cents «nègres de l'ancienne force armée de la Colonie, répandus dans les ateliers de la Grande-Terre. Ces hommes ont échappé en grande partie à toutes les mesures qui ont été prises pour les reconnaître et les faire enlever ${ }^{99}$. » Il n'est pas étonnant que la révolte ait lieu à Sainte-Anne. Cette commune a été le théâtre des conflits les plus violents entre patriotes et aristocrates en 1791, puis entre fédérés et sans-culottes en 1793. Ces affrontements peuvent se résumer dans la confrontation entre grands propriétaires et leurs opposants ${ }^{100}$, source probable de rancunes tenaces.

49 Le second problème des émigrés de retour est la conséquence directe des faits précédents : la diminution du nombre d'esclaves. La répression de la rébellion a entrâné des morts et des déportations d'esclaves devenus soldats. Depuis qu'il a pris le commandement de l'armée après la mort de Richepance en septembre 1802, Ménard estime que "plus de 2500 rebelles ont été ou pris ou tués, ou se sont rendus sur les habitations ou se sont pendus de désespoir dans les bois ${ }^{101}$ ». Ces morts s'ajoutent à ceux des combats de mai 1802 (au moins 1500), à ceux de la répression de juin à août, et à 2600 soldats de couleur déportés. Les recensements de la population de 1802 et de 1803 révèlent en outre une diminution de la population de 3044 individus ${ }^{102}$. Il pourrait s'agir $\mathrm{du}$ nombre des pertes civiles survenues lors de la répression, les recensements ne comptant pas les militaires. La main-d'œuvre disponible a donc diminué. En outre, des cultivateurs ont changé de plantation pendant la liberté générale. Louis Desmaisonneuve fait ainsi un procès à ses neveux, car il soutient qu'un «nombre considérable de nègres qui sont sur l'habitation [Gillet]» lui appartiennent. Finalement, le 15 octobre 1806, Desmaisonneuve et ses neveux décident de mettre fin à «ce procès qui ne peut que les entraîner dans des frais énormes et entretenir entre eux une mésintelligence qu'ils ont le désir d'éteindre ». Il finit ainsi par renoncer aux esclaves de l'habitation Gillet qu'il réclamait et en fait cession à ses neveux ${ }^{103}$. 
50 Le troisième problème est lié à l'endettement des propriétaires de retour et au mauvais état des habitations récupérées. Les émigrés se sont considérablement endettés, pendant leur séjour en dehors de la Guadeloupe, auprès des maisons de commerce avec lesquelles ils traitaient ou bien auprès d'amis et parents. Cet état d'endettement est supérieur à celui que les habitants-propriétaires ont connu avant la Révolution. Celui-ci était une caractéristique des propriétaires de manufacture à sucre sous l'Ancien Régime, lié aux modalités égalitaires de transmission des biens fonciers qui obligeaient celui qui voulait posséder la plantation à rembourser en valeur ses cohéritiers. L'endettement des manufacturiers du sucre est très supérieur à celui des propriétaires d'habitations secondaires restés à l'Anse-Bertrand pendant la période de la liberté générale. Alors que, pour les habitants-sucriers, le taux d'endettement varie de 15 à $120 \%$, celui des petits habitants est modulé entre 0 et $18 \%$.

51 Face à ces difficultés, certains décident de vendre. C'est le cas de Pierre Clair Ruillier Duclerc (1741-1811), propriétaire de la totalité d'une habitation, dite Duclerc, à l'AnseBertrand, de la moitié d'une autre au Port-Louis avec les enfants de son fils Pierre Germain Ruillier Duclerc (né en 1760 et mort à une date inconnue), et du quart d'une autre sucrerie, l'habitation dite René à Anse, dont un autre quart appartenait à son petitfils Étienne Duclerc (1763-1820), et la moitié à son neveu René Ruillier (1766-1821). Pierre Clair Ruillier Duclerc «a suivi la marche que les lois indiquent et notamment la déclaration du roi de 1726 qui défend le démembrement des sucreries ». À la suite d'une délibération de famille du 14 février 1805, il décide de vendre les trois habitations pour payer ses dettes et régler les frais de successions ${ }^{104}$. Le 24 avril 1805, il est procédé à la vente de l'habitation sucrerie appelée Duclerc du Port-Louis, achetée précédemment à M. Barbotteau. Elle reste dans la famille, car elle est vendue à deux de ses fils, Pierre Guillaume Ruillier Beauport (1773-1829) et Nicolas Ruillier Saint-Clair (1781-1812) ${ }^{105}$. Les habitations déjà en difficulté avant la Révolution voient donc leur situation se détériorer. Certains propriétaires de retour n'ont ni les moyens financiers de les réparer, faute de fonds, ni les moyens humains de les exploiter, par manque d'esclaves.

52 C'est d'ailleurs le cas de l'habitation Desmaisonneuve, appartenant à Louis Paul Gillet Desmaisonneuve, déjà évoquée plus haut. L'exploitation est encore qualifiée de sucrerie en 1796, mais sa main-d'œuvre est réduite à 33 cultivateurs. Le 14 avril 1807, elle est décrite comme " n'ayant pas les bâtiments nécessaires à la fabrication du sucre, et en une certaine quantité d'esclaves dont il ne peut dire au juste le nombre en ce moment ». Elle est composée de 100 carrés de terre plantés en cannes par M. Cadou ${ }^{106}$. En 1819, à la mort de Louis Paul Gillet Desmaisonneuve, la situation a empiré. Les biens étant toujours "grevés d'une masse de dettes que l'on ne pouvait payer sans aliéner tout ou grande partie des immeubles appartenant aux dites succession et communauté et même une partie des esclaves attachés, les parents et amis des mineurs de Maisonneuve réunis en conseil de famille et consultés à cet égard » décident de vendre 83 des 125 carrés de terre (30 $015 £$ ) et 24 des 42 esclaves ( $42700 £$ ) à Laurent Cadou. L'acquéreur s'engage à payer les $65935 £$ de dettes dues notamment aux sieurs Massiou, Pierre Angeron, Ardène et Guerry ${ }^{107}$. Ces derniers sont respectivement officier de santé, négociants et planteur. La période révolutionnaire a aggravé la situation de l'habitation Desmaisonneuve, que son propriétaire n'a pas réussi à améliorer pendant la Restauration. Ces cas particuliers montrent les difficultés des propriétaires de retour d'exil. Si l'endettement est quasigénéral, la plupart des émigrés conservent leurs propriétés. Lorsque celles-ci sont vendues, elles le sont bien souvent à des membres de la famille. 
53 La Guadeloupe se caractérise par un départ massif, sur le temps de la reconquête républicaine (juin-décembre 1794), de la catégorie la plus riche des planteurs, mais aussi par leur retour tout aussi massif sous le régime consulaire. Contrairement à la métropole, les biens des émigrés ne sont pas vendus. Les autorités coloniales estiment certainement qu'il n'y a pas d'acheteurs potentiels susceptibles d'acquérir les habitations à leur valeur réelle et préfèrent donc en assurer la gestion directement (1794-1798) ou les louer (1798-1802). Ce choix montre que, à la fois localement, mais aussi au niveau de la métropole, il y a une volonté de ne pas brader ces terres dont la valeur dépend essentiellement des bras qui les cultivent et de la possibilité d'exportation des denrées coloniales. La liberté générale, malgré les règlements de culture qui contraignent les esclaves à continuer à travailler sur les habitations de leurs anciens maîtres, crée un doute sur la possibilité de recourir au travail forcé des anciens esclaves. La guerre avec la Grande-Bretagne limite fortement l'exportation du sucre et du café. Elle engendre par conséquent une dépréciation des terres. La plupart des planteurs restés en Guadeloupe sont des petits et moyens propriétaires qui n'ont pas les capitaux suffisants pour acheter les manufactures à sucre à leur valeur antérieure à 1789. D'autre part, ces habitations confisquées permettent à la colonie d'avoir des revenus. En effet, avant la guerre, l'essentiel des revenus de la colonie provient des droits sur les entrées et sorties des marchandises. L'effondrement du commerce a donc tari cette ressource pour les finances de la colonie.

Le retour des émigrés se fait dans le cadre autoritaire du régime consulaire et s'accompagne du rétablissement de l'esclavage et du préjugé de couleur. À leur retour, les planteurs retrouvent l'intégralité de leurs terres, mais sont considérablement endettés. Pendant leur émigration, les planteurs ont continué à s'endetter, non plus seulement auprès de leurs familles ou de négociants, mais aussi de prêteurs situés dans les lieux d'exil.

55 À la différence de Saint-Domingue, où la totalité des planteurs partent massivement à la fin de 1803, en Guadeloupe, les émigrés sont revenus. Les sucreries de Guadeloupe connaissent ainsi une fragilité économique qui se poursuit tout au long du XIX $\mathrm{x}^{\mathrm{e}}$ siècle. Après la crise sucrière de 1884 , elles vont être rachetées pour la plupart par des sociétés de la métropole ${ }^{108}$. Ceci constitue une grande différence avec la Martinique où, du fait de l'occupation britannique de 1794 à 1802, frein à l'émigration, les propriétaires blancs créoles ont pu garder la mainmise sur le foncier jusqu'à nos jours.

\section{NOTES}

1. Gabriel DEBIEN, « De Saint-Domingue à Cuba avec une famille de réfugiés, les Tornézy : 1800-1809 ", Revue de la Faculté d'ethnologie de Port-au-Prince, nº 8, 1964, p. 7-31; Philip WRIGHT, Gabriel DEBIEN, « Les colons de Saint-Domingue passés à la Jamaïque (1792-1835) ", Bulletin de la Société d'histoire de la Guadeloupe, 4e trimestre, n² 26, 1975 ; Gabriel DEBIEN, "Réfugiés de Saint-Domingue expulsés de la Havane en 1809 », Notes d'Histoire coloniale, $\mathrm{n}^{\circ}$ 209, 1978, p. 555-610; Gabriel Debien, Les colons de Saint-Domingue réfugiés à la Louisiane, 
1792-1804, La Fayette, Revue de la Louisiane, 1981-1982 ; Marcel GRANDIÈRE, « Les réfugiés et les déportés des Antilles à Nantes sous la Révolution ", Bulletin de la Société d'histoire de la Guadeloupe, $\mathrm{n}^{\text {os }} 33-34,3^{\mathrm{e}}$ et $4^{\mathrm{e}}$ trimestre 1977, p. 3-171; Claude AUGUSTE et Marcel AUGUSTE, Les déportés de Saint-Domingue : contribution à l'histoire de l'expédition française de SaintDomingue (1802-1803), Québec, Éditions Noaman, 1979 ; P. LACHANCE, « Les réfugiés de SaintDomingue à la Nouvelle-Orléans : leur impact à court et long terme ", dans M. Hector (dir.), La Révolution française et Haïti, filiations, ruptures, nouvelles dimensions, 1995, p. 90-108 : P. LACHANCE, «Les vaincus de la Révolution haïtienne en quête d'un refuge de SaintDomingue à Cuba (1803), de Cuba à la Nouvelle-Orléans (1809) ", RSHHGG, vol. 38, n 126, 1980, p. 15-30 ; D. GEGGUS, « The Exile of the 1791 Slave Leaders: Spain's Resettlement of Its Black Auxiliary Troops », Journal of Haitian Studies, no 8-2, 2002, p. 52-67 ; J. DE CAUNA , "La diaspora des colons de Saint-Domingue et le monde créole : le cas de la Jamaïque », Revue Française d'Histoire d'Outre-Mer, n 304, 2004, p. 333-359 ; J.-C. BENZAKEN, « Le refus de l'abolition: les colons français réfugiés aux États-Unis et l'organisation des fêtes révolutionnaires ", dans M. Dorigny (dir.), Esclavage, résistances et abolitions, Paris, CTHS, 1999, p. 235-251. A. RENAULT, D’une île rebelle à une île fidèle, les Français de Santiago de Cuba (1791-1825), Mont-Saint-Aignan, Publications des Universités de Rouen et du Havre, 2012.

2. L. ABÉNON et M. DAUPHITE, Les Guadeloupéens réfugiés à Saint-Pierre de 1794 à 1796, Carbet, Éd. Centre d'Art Musée Paul Gauguin, 1990 ; F. MONFRET, Émigrés et autres réfugiés de la Guadeloupe vers les petites îles de la Caraibe, et tout particulièrement la Martinique, entre 1789 et 1802 : les départs, mémoire de DEA, université des Antilles-Guyane, 1993.

3. ANOM, DPPC G1/500-502. États nominatifs des citoyens de tout âge et de tout sexe au 1 er vendémiaire an V [22 septembre 1796] ; ANOM, DPPC G1/503-504. États nominatifs des citoyens de tout âge et de tout sexe au $1^{\mathrm{er}}$ vendémiaire an VI [22 septembre 1797].

4. A. LACOUR, Histoire de la Guadeloupe, Basse-Terre, 1857-1858, tomes 2 et 3.

5. Cette correspondance est conservée aux Archives Nationales d'Outre-Mer (ANOM) à Aix-en-Provence dans la série C7A.

6. ANOM, col. C7A 47, fo 5-6. Lettre des commissaires délégués écrite peu après l'arrivée de Lebas. s. d. Ce dernier arrive en Guadeloupe le 6 janvier 1795.

7. ANOM, DPPC G1/513. Listes des hommes suppliciés au Port-de-la-Liberté [Pointe-àPitre] et au Gosier.

8. A. LACOUR, op. cit., tome 2, p. 158.

9. AN DXXV 125, dossier 992, pièce 9. Tableau nominatif des personnes prévenues de délits contre-révolutionnaires, par leur présence ou agence, sous les ordres du gouverneur (le traître d'Arrot), à l'époque du mois de décembre 1792, dans les camps Saint-Jean, Picard et Darboussier, au siège de la ville Pointe-à-Pitre, et dans l'enlèvement et pillage de la gabarre La Bienvenue, à Saint-Christophe, sous les ordres de l'infâme Malvault, capitaine de La Calypso, chargé en chef de cette expédition avouée par l'assemblée coloniale rebelle, au mois d'octobre de la même année 1792, imprimé le 21 ventôse an II [11 mars 1794]. Document reproduit dans A. LAcour, op. cit., tome 2, p. 477-486.

10. A. LACOUR, op. cit., tome 2, p. 338-339.

11. ANOM, COL. C7A 49, fo 138-143. «Coup d'œil sur la Guadeloupe » en l'an V (1797) de Thouluyre Mahé. 
12. ANOM, DPPC NOT. GUA Lasalinière. Liquidation et partage de la société Papin frères et sœurs, 13 et 14 mars 1806.

13. F. RÉGENT, Esclavage, métissage, liberté, La Révolution française en Guadeloupe 1789-1802, Paris, Grasset, 2004, p. 254-266.

14. ANOM, DPPC G1 497. Liste des émigrés de la Guadeloupe en l'an IV. ANOM, DPPC G1 513. Liste des réfugiés et des émigrés de la Guadeloupe. Ministère des affaires étrangères, Centre des archives diplomatiques de Nantes, Guadeloupe, Correspondance volume 2. Liste des déportés de la Guadeloupe.

15. ANOM, DPPC G1 513. Liste des réfugiés et des émigrés de la Guadeloupe. Personnes émigrées de Goyave au 10 thermidor an IV [28 juillet 1796].

16. ANOM DPPC G1 497. Recensement de 1790.

17. ANOM DPPC G1 504. Tableau récapitulatif de la population de la Guadeloupe, s. d. [an IV].

18. De nombreux républicains ont été déportés à la suite de la conquête britannique en avril 1794.

19. ANOM DPPC G1/497. Liste des émigrés de la Guadeloupe ( $\left.{ }^{\circ} 61\right)$, brumaire an IV [novembre 1795].

Mes remerciements à Bernadette et Philippe Rossignol qui m'ont généreusement transmis leur liste d'émigrés que nous avons mise en base de données.

20. ANOM COL F3 237 Événements historiques Guadeloupe. Lettre de Victor Hugues au président de la Convention nationale du 26 frimaire an III [16 décembre 1794] du Port-dela-Liberté.

21. ANOM COL. C7A $85 \mathrm{~N}^{\circ} 27$. Tableau comparatif de la culture au $1^{\mathrm{er}}$ vendémiaire an VIII [23 septembre 1799] et au $1^{\text {er }}$ nivôse an IX [22 décembre 1800], daté de pluviôse an IX [21 janvier-19 février 1801].

22. ANOM DPPC G1 500 à 504. Ce tableau a été effectué à partir de nos dépouillements du recensement de la population de la Guadeloupe pour les communes du Nord de la GrandeTerre (Anse-Bertrand, Petit-Canal, Port-Louis) de la côte sous le vent (Bouillante, Deshaies, Pointe-Noire, Vieux-Habitants), de Basse-Terre, Trois-Rivières et Capesterre, soit dix des vingt-huit communes. Notre échantillon comprend 129 sucreries, 356 caféières, 139 cotonneries et 81 habitations vivrières.

23. ANOM DPPC NOT GUA Lasalinière. Acte du 24 février 1806, liquidation de la succession de Veuve Vannier Desbonnes des droits de son fils et de sa première épouse et de leur petit fils.

24. ANOM DPPC G1 513. État des personnes absentes de la commune de l'Anse-Bertrand, 17 brumaire an IV [8 novembre 1795]. Sur cette liste figure Gautonne et sa famille, sans indication du nombre.

25. ANOM DPPC G1513. Liste des émigrés de la commune d'Anse-Bertrand au 26 thermidor an IV [13 août 1796].

26. ANOM DPPC NOT. GUA, Pénicaut. Acte de société pour le commerce et transport de denrées entre Jacques Pierre Ruillier fils, domicilié à Anse-Bertrand et Etienne Ruillier Duclerc fils, négociant, à Pointe-à-Pitre, 20 germinal an XII [10 avril 1804].

27. GHC, nº 77, décembre 1995, p. 1494.

28. A. RENAULT, op. cit., p. 51. 
29. M. POLFLIET, Émigration et politisation : les Français de New York et La Nouvelle-Orléans dans la première moitié $d u x^{e}$ siècle (1803-1860), thèse d'histoire sous la direction de Silvia Marzagalli, Université de Nice, 2013).

30. ANOM DPPC NOT. GUA Pénicaut. Acte du 7 thermidor an 12 [26 juillet 1804]. Transaction Perrine Andreze Douillard la Bertaudière veuve en $1^{\mathrm{e}}$ noces de Guillaume Paviot et en $2^{\mathrm{e}}$ de Henry Gaugy.

31. ANOM DPPC NOT. GUA Pénicaut. Acte du 14 frimaire an 13 [5 décembre 1804]. Inventaire de la succession Gaugy.

32. Collection Baudouin. Décret qui ordonne la confiscation \& la vente des biens des Émigrés du 27 juillet 1792.

Ce décret est composé d'un article unique: "L'Assemblée Nationale décrète la confiscation \& la vente au profit de la Nation de tous les biens mobiliers \& immobiliers des Emigrés ».

33. ADG NOT Mollenthiel. Minute $n^{\circ} 12$ du 18 mai 1793, inventaire et séquestration des biens de Mallevault et de Pinel Dumanoir; ADG NOT Jaille. Minute du 14 juin 1793, inventaire et séquestration des biens d'Hurault de Gondrecourt ; minute du 17 juin 1793, séquestration des biens du baron de Bragelogne ; minute du 18 juin 1793, séquestration des biens d'Hercule Duqueruy.

34. ADG NOT Damaret. Minute $n^{\circ} 84$ du 8 octobre 1792. Recollement de l'inventaire des biens des Frères de la Charité. Les biens de l'ordre appartiennent désormais à la colonie ; ADG NOT Valeau. Minute $\mathrm{n}^{\circ} 2$ du 15 janvier 1793. Inventaire de l'habitation séquestrée Dolé, appartenant auparavant aux Carmes.

35. Collection Baudouin. Décret relatif à la vente des biens des émigrés dans les Colonies françaises, 25 Août 1792.

36. ANOM DPPC G1 513. Arrêté de Victor Hugues, Pointe-à-Pitre, le 25 messidor an II [13 juillet 1794].

37. ANOM COL. C7A 47, fo 36 . Arrêté du 2 frimaire an III [22 novembre 1794] du commissaire civil Victor Hugues.

38. ANOM COL F3 237. Evénements historiques Guadeloupe. Lettre de Victor Hugues au président de la Convention nationale du 26 frimaire an III [16 décembre 1794] du Port-dela-Liberté.

39. ANOM COL. C7A 49, fo 176 . Etat comparatif de la production des habitations des émigrés en 1789 et l'an $\mathrm{V}$.

40. ANOM COL. C7A 85, $\mathrm{n}^{\circ} 27$. Tableau comparatif de la culture au $1^{\text {er }}$ vendémiaire an VIII [23 septembre 1799] et au $1^{\mathrm{er}}$ nivôse an IX [22 décembre 1800] (pluviôse an IX [21 janvier-19 février 1801].

41. ANOM COL. C7A 53, fo 297 . Récapitulation générale des propriétés nationales de Guadeloupe au 26 vendémiaire an IX [18 octobre 1800].

42. A. LACOUR, op. cit., tome 2, p. 415-416.

43. A. LACOUR, op. cit., tome 2, p. 368.

44. Desfourneaux, successeur de Victor Hugues, administre la Guadeloupe de 1798 à 1799.

45. Jeannet, Laveaux et Baco, successeurs de Desfourneaux administrent la Guadeloupe de 1799 à 1801.

46. Ibid., p. 383. 
47. ANOM COL. C7A. Ordonnances, proclamations, arrêtés, circulaires (1788-1811). Arrêté du 26 ventôse an III (16 mars 1795) des commissaires délégués par la Convention aux Iles du Vent du Port-de-la-Liberté.

48. Éric TEYSSIER, «La vente des biens nationaux et la question agraire, aspects législatifs et politiques, 1789-1795 ", Rives nord-méditerranéennes, $\mathrm{n}^{\circ}$ 5, 2000, p. 45-62.

49. A. LACOUR, op. cit., tome 2, p. 387.

50. ANOM 1DPPC Registre d'état civil d'Anse-Bertrand. Acte de mariage du 18 novembre 1796.

51. A. LACOUR, op. cit., tome 2, p. 447-448.

52. ADG Registre des délibérations de l'administration municipale de Sainte-Anne, 19 floréal an III [8 mai 1795]. Réunion du conseil municipal avec le commissaire délégué.

53. A. LACOUR, op. cit., tome 2, p. 387.

54. ADG Registre des délibérations de l'administration municipale de Sainte-Anne, 29 floréal an III [18 mai 1795].

55. ADG Registre des délibérations de l'administration municipale de Sainte-Anne, 19 floréal an III [8 mai 1795]. Réunion du conseil municipal avec le commissaire délégué.

56. ANOM COL. C7A. Ordonnances, proclamations, arrêtés, circulaires (1788-1811). Arrêté du 26 ventôse an III (16 mars 1795) des commissaires délégués par la Convention aux Iles du Vent du Port-de-la-Liberté.

57. A. LACOUR, op. cit., tome 3, p. 23-24.

58. Loi relative aux fonds nécessaires pour les dépenses générales, ordinaires et extraordinaires de l'an VI, etc., qui ordonne le remboursement de la dette publique, deux tiers en bons au porteur, et un tiers en inscriptions sur le grand livre, et fixe l'emploi des bons en acquisition de domaines nationaux du 9 vendémiaire an VI [30 septembre 1797], citée dans ALLIER et CERCLET, Manuel de l'émigré, Paris, Chez Delaforet, 1825, p. 219.

59. Loi concernant l'organisation constitutionnelle des colonies $\left(1^{\mathrm{er}}\right.$ janvier $1798-12$ nivôse an VI), citée dans ALLIER et CERCLET, op. cit., p. 240-241.

60. ANOM COL. C7A 53, fo 229-230. Arrêté du 29 messidor an VII [17 juillet 1799] du Portde-la-Liberté (Pointe-à-Pitre) sur les fermes inséré dans le compte rendu ou rapport fait au ministre de la marine et des colonies par Desfourneaux, Paris, 27 pluviôse an VIII [16 février 1800].

61. ADG NOTARIAT SAINT-MARTIN. Minute $\mathrm{n}^{\circ} 21$, convention entre Jean Joseph Mamon et Cyprien Dugommier pour la gestion d'une habitation située aux Trois-Rivières le 11 thermidor an VIII [30 juillet 1800].

62. ANOM COL. C7A 52, fo 7. Adjudication d'une habitation nationale pour 89 milliers de sucre au citoyen Robin, le 7 thermidor an VII (5 juillet 1799).

63. ADG NOTARIAT DUPUCH. Minute du 5 thermidor an VII [3 juillet 1799], formation d'une société entre les citoyens François Robert et Guillaume Costet pour exploiter l'habitation Fond Cacao.

64. ANOM DPPC Etat-civil Anse-Bertrand. Acte du $1^{\text {er }}$ mai 1800, mentionne Desbonne Vannier, locataire de l'habitation René Ruillier.

65. ADG NOTARIAT SAINT-MARTIN. Minute $\mathrm{n}^{\circ} 21$, convention entre Jean Joseph Mamon et Cyprien Dugommier pour la gestion d'une habitation située aux Trois-Rivières le 11 thermidor an VIII [30 juillet 1800]. 
66. ADG NOTARIAT SAINT-MARTIN. Minute $n^{\circ} 31$, dépôt d'un paquet cacheté par Jean

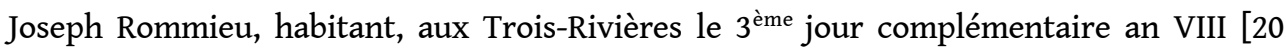
septembre 1800].

67. ANOM COL. C7A 52, fo115-117. Lettre de Baco du 4 thermidor an VIII [23 juillet 1800], s. 1.

68. ANOM COL. C7A 82 pièce 44 . Rapport du ministre de la marine et des colonies aux consuls du 28 pluviôse an VIII [17 février 1800].

69. ANOM COL. C7A 52, f ${ }^{\circ} 170-173$. Arrêté des agents du Directoire du 27 pluviôse an VIII (17 février 1800) concernant les fermages.

70. M. Vovelle, «Biens nationaux et politisation paysanne pendant la Révolution française ", dans Pratiques et cultures politiques dans la France contemporaine (hommage à Raymond Huard), Montpellier, Université Paul Valéry, 1995, p. 35-47.

71. ANOM G1 497. Recensement de la population de 1790.

ANOM COL. C7A 85. Rapport des agents des consuls Baco, Jeannet et Laveaux au ministre de la marine et des colonies. Etat comparatif de la culture aux $1^{\mathrm{er}}$ vendémiaire an VIII et 1 er nivôse an IX (23 septembre 1799-22 décembre 1800), paraphé le 20 pluviôse an IX (9 février 1801).

72. ANOM COL. C7A 51, fo 204 . Aperçu des revenus et des dépenses des habitations nationales

73. C. SCHNAKENBOURG, «Statistiques pour l'histoire de l'économie de plantation en Guadeloupe et en Martinique », Bulletin de la société d'Histoire de la Guadeloupe, $1^{\mathrm{er}}$ trimestre 1977, n 31, p. 3-121.

74. ANOM COL. C7A 52, fo 20-23. Lettre des agents au ministre, du Port-de-la-Liberté, le 5 pluviôse an VIII [25 janvier 1800].

75. ANOM COL. C7A 52, fo $4-5$. Lettre des agents au ministre le 4 pluviôse an VIII [24 janvier 1800] du Port-de-la-Liberté [Pointe-à-Pitre].

76. ANOM COL. C7A 52, fo 208. Circulaire des agents des consuls du $1^{\text {er }}$ brumaire an IX [ 23 octobre 1800] de Basse-Terre.

77. ANOM COL. C7A 52, fo $121-127$. Lettre des agents du Directoire au ministre, le 12 thermidor an VIII [31 juillet 1800] ; ANOM COL. C7A 54, fo30. Circulaire aux commissaires du gouvernement dans les différents cantons du gouvernement pour le paiement du cinquième terme des baux, le 7 pluviôse an IX [27 janvier 1801].

ANOM COL. C7A 54, fo 219. Circulaire du 23 germinal an IX [13 avril 1801] des agents du Directoire aux commissaires du gouvernement pour le paiement du sixième terme des baux. ANOM COL. C7A 55, fo 33. Circulaire de Lacrosse aux commissaires de gouvernement les pressant de faire rentrer les fermages, le 25 prairial an IX [14 juin 1801].

78. A. LACOUR, op. cit., tome 2, p. 411-413.

79. Ibid., p. 383.

80. ANOM COL. C7A 55, fo 246 . Lettre de Duny du 19 germinal an IX [9 avril 1801], de Portde-la-Liberté [Pointe-à-Pitre].

81. ANOM COL. C7A 55, fo 279-282.Abrégé historique des événements arrivés pendant le mois de prairial an IX [mai-juin 1801].

82. A. LACOUR, op. cit., tome 3, p. 118. 
83. E. DE WARESQUIEL, "Joseph Fouché et la question de l'amnistie des émigrés (1799-1802) », Annales historiques de la Révolution française, $\mathrm{n}^{\circ}$ 372, 2013, p. 105-120.

84. ALLIER et CERCLET, op. cit., p. 267-270.

85. A. LACOUR, op. cit., tome 3, p. 119.

86. Ibid., p. 125-126.

87. ALLIER et CERCLET, op. cit., p. 177.

88. A. LACOUR, op. cit., tome 3, p. 357 et 436.

89. ALLIER et CERCLET, op. cit., p. 284.

90. E. BOYER-PEYRELEAU, Les Antilles françaises particulièrement la Guadeloupe, depuis leur découverte jusqu'au $1^{\text {er }}$ novembre 1825, L'Advocat, 1825, tome 1, p. 401-402.

91. A. LACOUR, op. cit., tome 3, p. 436. ; ALLIER et CERCLET, op. cit., p. XLIX. Arrêté concernant le retour des propriétaires blancs dans les colonies.

92. A. LACOUR, op. cit., tome 3, p. 306.

93. ANOM DPPC G1/513. Habitants absents de la colonie par congé, émigrés et de retour dans la colonie : états nominatifs (1796/1803) Personnes rentrées dans la colonie et ayant prêté serment entre les mains de l'autorité coloniale : Guadeloupe, propriétaires réfugiés à la Martinique, 19 thermidor an X [7 août 1802]; Guadeloupe, émigrés (1er vendémiaire an XI [23 septembre 1802]) ; Pointe-à-Pitre (an X).

94. ANOM DDPPC NOT GUA Lasalinière. Minute des 13 et 14 mars 1806, liquidation et partage de la société Papin frères et sœurs.

95. Bibliothèque municipale de Lyon. Ménard, Mémoire du général de brigade Ménard sur ce qui s'est passé à la Guadeloupe le 28 germinal de l'an 11, s. l., s. n., après 1805, p. 33.

96. A lacour, op. cit., tome 3, p. 405.

97. Ibid., p. 406.

98. Ibid., p. 27

99. Ibid., p. 25.

100. F. RÉGENT, Esclavage, métissage liberté, op. cit., p. 226, 256-261.

101. Ibid., p. 3-4.

102. ANOM COL. C7A 85 et E. BOYER-PEYRELEAU, op. cit., tome II, in fine, tableau $n^{\circ} 3$.

103. ANOM DPPC NOT GUA Pénicaut. Minute du 15 octobre 1806.

104. ANOM DDPPC NOT GUA Lasalinière. Minute $n^{\circ} 30$ du 25 pluviôse an 13 [14 février 1805], délibération qui règle les clauses et les conditions de la vente des habitations dites Duclerc.

105. ANOM DDPPC NOT GUA Lasalinière. Minute $n^{\circ} 46$ du 4 Floréal an 13 [24 avril 1805], vente de l'habitation Duclerc à Port-Louis.

106. ANOM DDPPC NOT GUA Lasalinière. Minute $\mathrm{n}^{\circ} 19$ du 14 avril 1807, contrat de mariage entre M. Louis Paul Gillet Desmaisonneuve, fils majeur de feu sieur Paul Jacques Gillet Desmaisonneuve et feue Charlotte Ruillier, et Mme Marie Louise Fletcher habitante à l'Anse-Bertrand, veuve de M. Marc Desbonne, fille majeure de feu sieur Nicolas Fletcher et feue Marie Françoise Ruillier.

107. ANOM DDPPC NOT GUA Lasalinière. Minute du 23 février 1819. 
108. C. SCHNAKENBOURG, Histoire de l'industrie sucrière en Guadeloupe, fluctuations et dépendances (1884-1946), Paris, L'Harmattan, 2009.

\section{RÉSUMÉS}

Entre 1794 et 1802, près de trois mille personnes fuient la Guadeloupe après la reconquête de l'île par les Républicains. Il s'agit de planteurs et des membres de leurs familles. Ce sont principalement des manufacturiers du sucre, les plus grands propriétaires de terres et d'esclaves de la colonie. Parallèlement à cette émigration, l'esclavage est aboli. Les propriétés abandonnées sont désormais gérées par les autorités républicaines. Elles vont avoir recours au système des séquestres, puis à la location. À la différence de la métropole, les biens des émigrés ne sont pas mis en vente. À leur retour, ils reprennent possession de leurs terres et de leurs esclaves, au moment du rétablissement de l'esclavage. Ils retrouvent des plantations en mauvais état et sont fortement endettés.

Between 1794 and 1802, nearly 3,000 people flee Guadeloupe, after the recapture of the island by the Republicans. These are the planters and their family members. They are mainly sugar producers, the largest landowners and slaves in the colony. Parallel to this emigration, slavery is abolished. The abandoned properties are now managed by the Republican authorities. They will use the escrow system and then leasing. Unlike the metropolis, the goods of the emigrants are not put on sale. On their return, they regain possession of their lands and their slaves, when slavery is re-established. They found themselves with plantations in poor condition and are heavily indebted.

INDEX

Keywords : Emigrants, Guadeloupe, Plantation, Abolition, Counter-Revolution

Mots-clés : Émigrés, Guadeloupe, Plantation, Abolition, Contre-révolution

\section{AUTEUR \\ FRÉDÉRIC RÉGENT}

IHMC

Université Paris 1 